\title{
Assessing Inhomogeneity in Sorted Samples of Single-Walled Carbon Nanotubes through Fluorescence and Variance Spectroscopy
}

\author{
Yara Kadria-Vili, ${ }^{\mathrm{a}, *}$ Stephen R. Sanchez, ${ }^{\mathrm{a}, *}$ Sergei M. Bachilo, ${ }^{\mathrm{a}, * *}$ \\ and R. Bruce Weisman ${ }^{\mathbf{a}, \mathbf{b}, * * *, \mathrm{z}}$ \\ ${ }^{a}$ Department of Chemistry and the Smalley-Curl Institute, Rice University, Houston, Texas 77005, USA \\ ${ }^{b}$ Department of Materials Science and NanoEngineering, Rice University, Houston, Texas 77005, USA
}

\begin{abstract}
Detailed spectroscopic analysis has been used to study the homogeneity of single-walled carbon nanotube fractions carefully prepared by nonlinear density gradient ultracentrifugation sorting. Two distinct colored bands containing $(6,5)$ enantiomers were subdivided into several extracted fractions that were separately diluted with sodium cholate surfactant and characterized by fluorescence, absorption, and variance spectroscopy. Values were measured for emission and absorption peak positions, Stokes shifts, emission peak widths, and emissive quantum yields. In addition, variance data were used to find relative emission per nanotube and to plot covariance slices representing homogeneous emission spectra. It was found that emission from SWCNTs within the upper enantiomer band shifts to shorter wavelengths with increasing depth in the centrifuge tube. In the lower enantiomer band such spectral shifts were not observed, but the emissive quantum yields decreased with depth. Variance analysis revealed spectral differences among SWCNTs within the same fraction of the same band. It is concluded that current methods for density gradient ultracentrifugation sorting produce samples that retain measurable structural and spectral inhomogeneities.

(C) The Author(s) 2017. Published by ECS. This is an open access article distributed under the terms of the Creative Commons Attribution 4.0 License (CC BY, http://creativecommons.org/licenses/by/4.0/), which permits unrestricted reuse of the work in any medium, provided the original work is properly cited. [DOI: 10.1149/2.0171706jss] All rights reserved.

(cc) BY
\end{abstract}

Manuscript submitted December 28, 2016; revised manuscript received January 16, 2017. Published January 27, 2017. This paper

is part of the JSS Focus Issue on Nanocarbons - In Memory of Sir Harry Kroto.

Single-walled carbon nanotubes (SWCNTs) are probably the best known and most intensely studied artificial nanomaterial. For some purposes, SWCNTs may be viewed as a single substance because all of them are tubular structures formed from covalently bonded, $\mathrm{sp}^{2}$-hybridized carbon atoms. However, every SWCNT has a specific crystalline structure defining a particular diameter and roll-up angle. ${ }^{1}$ Each such structural species is uniquely labeled by a pair of integers, $(n, m)$, that designate how a graphene sheet can be rolled up to generate that SWCNT. In addition to having specific physical structures, the different $(n, m)$ species also display distinct vibrational frequencies, electronic energy levels, transport properties, optical spectra, strain energies, redox potentials, and chemical reactivities. They should therefore be considered separate chemical substances.

Unfortunately, all current methods for growing SWCNTs in practical quantities produce a range of $(n, m)$ structures and thus give samples with highly inhomogeneous properties. Such mixtures are less useful for many refined applications and also pose challenges in basic research studies. The sorting of mixed SWCNT samples into structurally pure fractions is therefore a major goal of the field. Purification involves not only fractionation by $(n, m)$ structure, but also the separation of individualized from aggregated SWCNTs and the removal of residual catalyst particles, amorphous carbon, and perhaps double- or multi-walled nanotubes. In addition, some sorting methods can separate the left- and right-handed enantiomeric forms of chiral SWCNTs and the water-filled from the empty forms of $(n, m)$ species. $^{2-6}$

Methods for reducing the extensive inhomogeneity of SWCNT samples must be supported by powerful characterization tools to guide and assess separation efforts. In our view, optical absorption and emission spectroscopy provide the best combination of speed, simplicity, and analytical power for characterizing the semiconducting SWCNT species that dominate most samples. ${ }^{7,8}$ We report here the use of detailed optical analysis, including a recently introduced advanced method called variance spectroscopy, ${ }^{9}$ to probe for residual inhomogeneities present in SWCNT fractions prepared by nonlinear

\footnotetext{
*Electrochemical Society Student Member.

***Electrochemical Society Member.

***EElectrochemical Society Fellow.

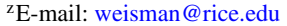

density gradient ultracentrifugation (NDGU). ${ }^{2}$ This is a refined sorting method that is selective enough to prepare enantiomeric forms of an individual $(n, m)$ species. Nevertheless, our results reveal unexpected inhomogeneities within carefully prepared NDGU samples. These findings suggest that the preparation of homogeneous bulk SWCNT samples remains a challenge for the nanotube research community.

\section{Experimental}

Sample preparation.-We prepared a sample of $(6,5)$ enantiomers through NDGU, using a method similar to that described previously. ${ }^{10}$ We first added a $5 \mathrm{mg}$ sample of raw HiPco SWCNTs produced in the Rice University reactor (batch 189.2 ) to $10 \mathrm{~mL}$ of $2 \%(\mathrm{w} / \mathrm{v}$ ) aqueous sodium cholate (SC) solution. The mixture was bath sonicated for $1 \mathrm{~h}$ and then tip sonicated at $7 \mathrm{~W}$ for $30 \mathrm{~min}$ while cooled in an ice bath. Finally, it was centrifuged to obtain a supernatant enriched in individualized nanotubes and depleted of bundles, carbonaceous impurities, and residual iron catalyst. To prepare a sample for injection into the density gradient separation, we added to this supernatant a solution of $60 \%$ (w/v) aqueous iodixanol (Sigma-Aldrich OptiPrep) containing $2 \% \mathrm{SC}$ and a smaller amount of sodium dodecyl sulfate (SDS). The resulting SWCNT suspension had a volume of $880 \mu \mathrm{L}$ and contained $2 \%$ SC, $0.2 \%$ SDS, and $25 \%$ iodixanol.

The iodixanol density gradient was formed by sequentially layering aqueous solutions with different iodixanol contents into a $5 \mathrm{~mL}$, $13 \mathrm{~mm}$ diameter Polyclear open-top ultracentrifuge tube. We prepared these solutions from an aqueous stock solution containing $30 \%$ iodixanol, $0.7 \%$ SC, and $0.175 \%$ SDS, which was diluted as needed with a solution having the same surfactant concentrations. The gradient medium was layered using a Pasteur pipette from bottom to top of the centrifuge tube in the order: $30 \%$ iodixanol $(500 \mu \mathrm{L}), 27.5 \%(420$ $\mu \mathrm{L}), 25 \%(660 \mu \mathrm{L}), 22.5 \%(660 \mu \mathrm{L}), 20 \%$ (660 $\mu \mathrm{L}), 17.5 \%(725$ $\mu \mathrm{L})$, and $15 \%(785 \mu \mathrm{L})$. Afterward, the centrifuge tube was placed at a 39 degree angle from vertical for $50 \mathrm{~min}$ to allow diffusional formation of a smooth nonlinear density gradient.

The final step in preparing the NDGU sample tube was injecting the SWCNT suspension described above into the density gradient at a depth corresponding to $25 \%$ iodixanol content. The tube was then ultracentrifuged for $22 \mathrm{~h}$ at $250000 \mathrm{~g}$ at a temperature of $20^{\circ} \mathrm{C}$. During 


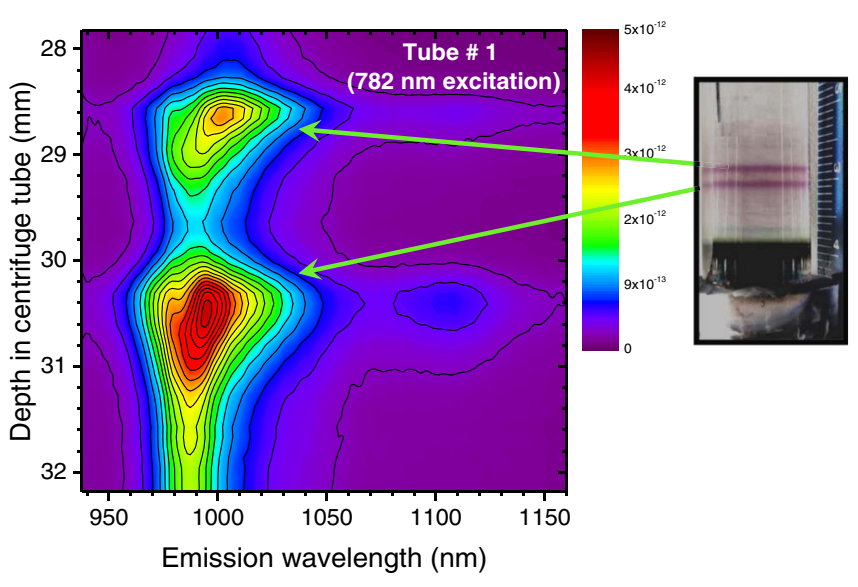

Figure 1. Right frame: photo of the NDGU-processed centrifuge tube with two pink bands. Left frame: Expanded contour plot of in situ emission spectra measured through the centrifuge tube wall as a function of depth coordinate. The excitation wavelength was $782 \mathrm{~nm}$. The two dominant features are enantiomers of $(6,5)$ SWCNTs.

centrifugation, nanotubes migrated to positions at which their buoyant densities matched those of the surrounding medium. This resulted in the formation of discrete layers enriched in a single nanotube species (see Figure S3). Our gradient was designed to give a shallow density gradient, and therefore high spatial separations, near the density of the two pink layers shown in Figure 1.

By using a prototype model NS3 NanoSpectralyzer (Applied NanoFluorescence) equipped with a vertical sample translator, we captured in situ near-IR fluorescence spectra as a function of depth in the tube following ultracentrifugation. We chose a vertical step size of $0.1 \mathrm{~mm}$ and an excitation wavelength of $782 \mathrm{~nm}$ to map the two pink bands separated by $\sim 2 \mathrm{~mm}$ (see Figures 1 and S1). The two bands were then delicately extracted under computer control with a custom-built precision fractionator. ${ }^{11}$ We collected four fractions from the upper band and three fractions from the lower band. Each was obtained by smoothly moving the collection needle in a horizontal spiral path as liquid was extracted at a rate of $20 \mu \mathrm{L} / \mathrm{min}$. The needle was then lowered by $0.4 \mathrm{~mm}$ for collection of the adjacent fraction. Once extracted, the $48 \mu \mathrm{L}$ fractions were diluted by a factor of six with $2 \%$ SC surfactant solution to displace other components from the SWCNT surface.

Bulk spectroscopy.-Bulk SWIR fluorescence spectra and visible and SWIR absorption spectra were acquired with a prototype model NS2 NanoSpectralyzer (Applied NanoFluorescence, LLC) using a $3 \mathrm{~mm}$ path length fused silica cuvette. Fluorescence excitation wavelengths were 642, 659, and $784 \mathrm{~nm}$. We measured absolute fluorescence quantum yields using LDS820 dye as a calibrated reference, with 642 and $659 \mathrm{~nm}$ excitation. ${ }^{12}$

Length analysis. - Length distributions of SWCNT fractions were measured using the diffusion-based LAND method, as described previously. ${ }^{13}$

Variance spectroscopy.-To measure variance spectra, we used a slightly modified version of an apparatus and method previously described in some detail..$^{9,14}$ Samples were held in a $100 \mu \mathrm{m}$ path length demountable fused silica cuvette that was translated stepwise to allow collection of emission spectra from 2000 different spatial regions. Excitation was provided by focused diode lasers emitting at $660 \mathrm{~nm}(82$ $\mathrm{mW}$, Power Technology, Inc.) or $784 \mathrm{~nm}$ ( $80 \mathrm{~mW}$, Vortran Laser Technology, Inc). The laser beams were collinearly combined using a 724 nm DRLP dichroic reflector (Omega Optical). To remove any stray SWIR emission from the lasers, the $660 \mathrm{~nm}$ beam was passed through two short pass filters (875 and $1100 \mathrm{~nm}$ cutoffs, Edmund Optics), and the $784 \mathrm{~nm}$ beam was cleaned by a band pass filter (780BP80, Newport Corp.). The collinear excitation beams were reflected by a dichroic mirror and focused onto the sample from above by a fused silica aspheric lens (EFL $=8.0 \mathrm{~mm}, \mathrm{NA}=0.63$, Edmund Optics). Emission was collected by that lens in epifluorescence geometry and passed through the dichroic mirror and an $850 \mathrm{~nm}$ long pass filter into a fiber collimator ( $\mathrm{f}=11 \mathrm{~mm}$, Thorlabs). Finally, a multimode optical fiber (50 $\mu \mathrm{m}$ core diameter, 0.39 NA, Thorlabs) transmitted the emission to an SWIR spectrometer with a 512 channel InGaAs detector thermoelectrically cooled to $-5^{\circ} \mathrm{C}$ (B\&WTek Sol 1.7). We used an integration time of 150-200 ms for all experiments. Mean spectra were calculated directly from the 2000 spatially independent spectra, whereas variance spectra were calculated after two corrections. First, within each spectral channel, individual points deviating more than $5 \sigma$ from the mean were omitted to account for fluorescence artifacts such as cuvette optical irregularities. Then smoothing by a local regression model using a $2^{\text {nd }}$ degree polynomial was applied to account for any time-dependent changes in the fluorescence. A similar approach was used for covariance analysis, except that the deviation threshold for neglecting individual points was set to $3 \sigma$ from the mean.

Numerical simulations.-We performed numerical simulations using MATLAB software (MathWorks). Emission profiles were generated from a recently developed photoluminescence model that includes four different emission features for each $(n, m)$ species. ${ }^{15}$ These were the main $\mathrm{E}_{11}$ transition and three side bands (300, 1068, and 1600 $\mathrm{cm}^{-1}$ below $\mathrm{E}_{11}$ ), whose relative amplitudes and widths are expressed relative to $\mathrm{E}_{11}$. The $\mathrm{E}_{11}$ transition was modeled with a Gaussian line shape, a Lorenztian line shape, or a Pearson IV function, while all sideband features were modeled as Gaussians for simplicity. For simulating variance data, we modeled fluctuations in emission intensities using random values following a normal distribution. The relative fluctuations in main and side bands were assumed to be identical. We calculated the mean, variance, and 2D covariance profiles directly from these fluctuations.

\section{Results and Discussion}

In situ spectra.-The right side of Figure 1 shows a photo of the NDGU-processed sample tube, in which the two prominent pink bands are the subject of our current study. We identify these bands as the enantiomeric forms of $(6,5)$ SWCNTs on the basis of prior research ${ }^{2,10,16-18}$ and the adjacent in situ spectral map showing their emission peaks near $980 \mathrm{~nm}$ in depth-resolved spectra induced by 782 $\mathrm{nm}$ excitation. As was discussed previously, the density-based separation of $(6,5)$ enantiomers is possible in certain surfactant mixtures of SC and SDS because the SC is chiral and has slightly different affinities for coating the two enantiomers. In the presence of an appropriate competing concentration of SDS cosurfactant, this results in enantiomers coated with different ratios of the two surfactants. The enantiomers with SDS-enriched surface coatings have a slightly greater buoyant density. During ultracentrifugation in a density gradient, they migrate to a position in the centrifuge tube where the surrounding density matches theirs, while the other enantiomer forms a band at a slightly higher position. Figure S3 illustrates this process. As described in Experimental Methods, the two bands were fractionated to give four extracted layers from the upper one and three extracted layers from the bottom one. Our goal is to use spectral measurements to explore SWCNT inhomogeneities between and within the two bands.

The spectral contour map in Figure 1 shows emission peaks that shift to shorter wavelengths with increasing depth in the centrifuge tube. However, SWCNT fluorescence spectra are particularly subject to distortions from the inner filter effect, in which some of the emitted light is absorbed in the sample before reaching the detector. ${ }^{19}$ SWCNT emission peaks overlap strongly with the intense $E_{11}$ absorption peaks and have small Stokes shifts. For samples that are not dilute enough to be optically thin, this can cause selective absorption of the shorter wavelength portions of emission spectra and shift the apparent emission peaks to longer wavelength. In fact, we have found 
that the SWCNT concentrations in the unextracted bands are 7 to $9 \mu \mathrm{g} / \mathrm{mL}$, giving peak $E_{11}$ absorbance values greater than 1.1 per $\mathrm{cm}$. This causes inner filter distortions and accounts for some of the position-dependent peak shifts seen in Figure 1. By analyzing absorption and emission spectra of the extracted fractions, we were able to estimate the effective inner filter distance as $1.2 \mathrm{~mm}$ and numerically correct for those distortions in the spectra (see Figures S4-S5). This correction blue-shifted the peaks in the upper band by ca. $10 \mathrm{~cm}^{-1}$ and sharpened them by ca. $48 \mathrm{~cm}^{-1}$ (see Figure S6). All further measurements were performed after diluting each extracted fraction by a factor of 6 with aqueous $2 \%$ SC. This dilution suppressed inner filter distortions. It also increased the ratio of SC to SDS concentrations to 62. This large excess, plus the higher affinity of SC for $(6,5)$ SWCNTs, ensured that any residual SDS was displaced from the SWCNTs.

Spectral comparison of upper and lower bands.-Before performing detailed comparisons of emission spectra from the extracted fractions, it was necessary to select which of our discrete excitation wavelengths gave the spectra with minimal contributions from $(n, m)$ impurities, i.e. species other than $(6,5)$. Variance spectroscopy provided an effective tool for this selection. As described previously, ${ }^{9}$ variance spectroscopy captures many fluorescence spectra from different small regions of a liquid sample that is uniform on the macroscopic scale. Because of the limited number of emissive particles (nanotubes) in each probed region, statistical differences in concentrations are observed as measurable differences in the spectra. We analyze the set of data at each wavelength to obtain $\bar{I}(\lambda)$, the mean spectrum (first moment), and $\sigma^{2}(\lambda)$, the variance spectrum (second moment). The ratio $N=\bar{I}^{2} / \sigma^{2}$ reveals the number of particles in the probed volume that contribute to the spectrum at each wavelength.

If all emission contributions are from only one $(n, m)$ species, $N$ is independent of wavelength and the normalized plots of $\bar{I}^{2}(\lambda)$ and $\sigma^{2}(\lambda)$ should be superimposable. However, if there are overlapping emission contributions from different $(n, m)$ species, then the variance data will exceed the squared mean at wavelengths of spectral overlap. As shown in Figure 2 (top), a sample fraction excited at $660 \mathrm{~nm}$ shows clear differences between the two plots in the wings of the main feature marked by the boxes. These reflect emission from small concentrations of $(8,3)$ and $(7,5)$ SWCNT impurities, which are excited more efficiently than $(6,5)$ at $660 \mathrm{~nm}$. However, the emission observed with $784 \mathrm{~nm}$ excitation is almost entirely from $(6,5)$, as can be seen by the very close agreement between the squared mean and variance spectra plotted in Figure 2 (bottom). We therefore chose to analyze only 784 $\mathrm{nm}$ excitation data to selectively study inhomogeneities among the $(6,5)$ nanotubes.

Normalized emission spectra from all seven extracted and diluted fractions are plotted in Figure 3. Emission peaks of the four upper band fractions show an average blue-shift of $38 \mathrm{~cm}^{-1}$ on dilution with $2 \%$ SC. Dilution also significantly sharpens the emission from upper band fractions, reducing their average full-width at half-maximum (fwhm) from $259 \mathrm{~cm}^{-1}$ (after inner filter correction) to $217 \mathrm{~cm}^{-1}$. We interpret these changes as reflecting a more uniform dielectric environment and greater isolation of the SWCNT surface from water through formation of a more complete surface coating of SC. By contrast, the lower band fractions show minimal peak shifts or sharpening on dilution. We find that the average peak widths are approximately 9 $\mathrm{cm}^{-1}$ smaller for the lower band fractions than upper band fractions. In addition, there is an average shift of ca. $12 \mathrm{~cm}^{-1}$ between the peak positions of diluted upper and lower fractions, with the upper fractions at lower frequency. Because the intrinsic electronic structures of the two enantiomers are identical, this difference must arise from a handedness-related difference in the packing density of SC on the nanotube surfaces. Absorption spectra measured from all seven extracted fractions are plotted in Figure 4 after baseline adjustment and normalization. The Stokes shifts, measured as the frequency difference between absorption and emission peaks, are approximately 16 $\mathrm{cm}^{-1}$ for both upper and lower band fractions. Our LAND measurements found an unexplained difference in average length between the

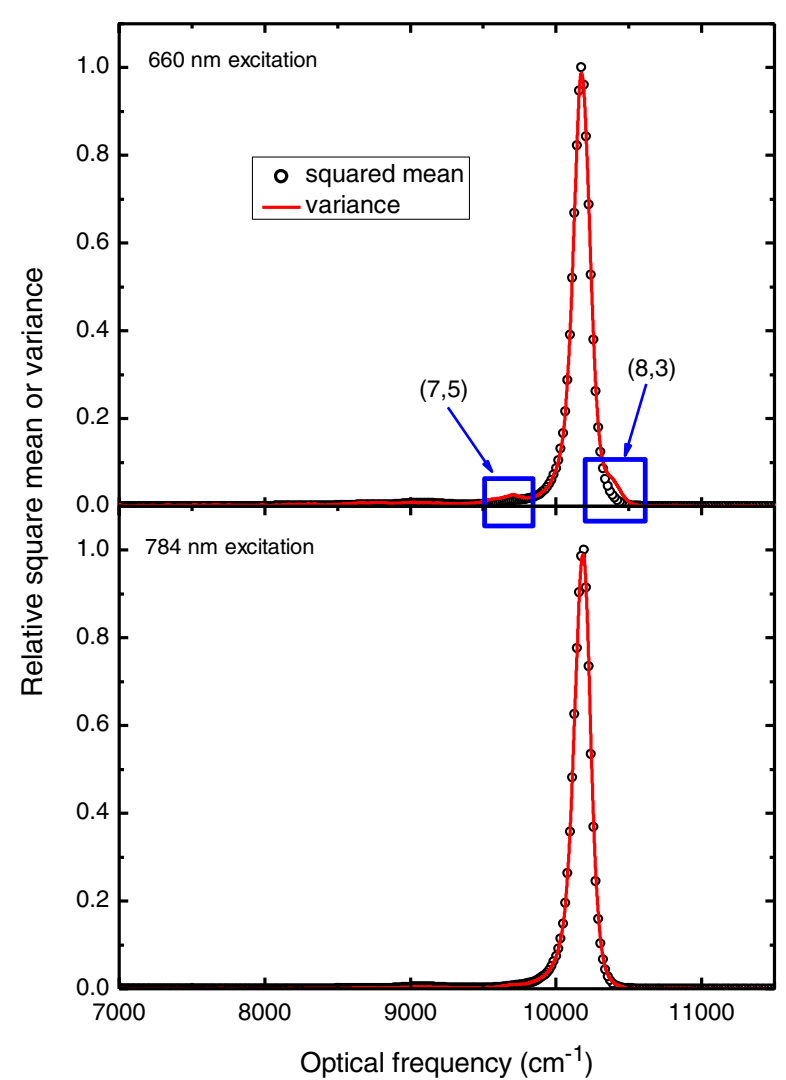

Figure 2. Comparisons of normalized squared mean (black circles) and variance (red curves) spectra of the fourth fraction of the upper NDGU band. Excitation wavelength was $660 \mathrm{~nm}$ for the top frame and $784 \mathrm{~nm}$ for the bottom frame. The blue boxes mark regions of mismatches between the spectra, attributed to minor impurities of $(7,5)$ and $(8,3)$ SWCNTs.

first fractions of the upper and lower bands: $475 \mathrm{~nm}$ for upper and 300 $\mathrm{nm}$ for lower. This represents a structural inhomogeneity between the bands, but it seems unlikely to be related to the small spectral differences described above.

Spectral comparison within bands. - The three fractions extracted from the lower band showed emission peak positions, widths, and

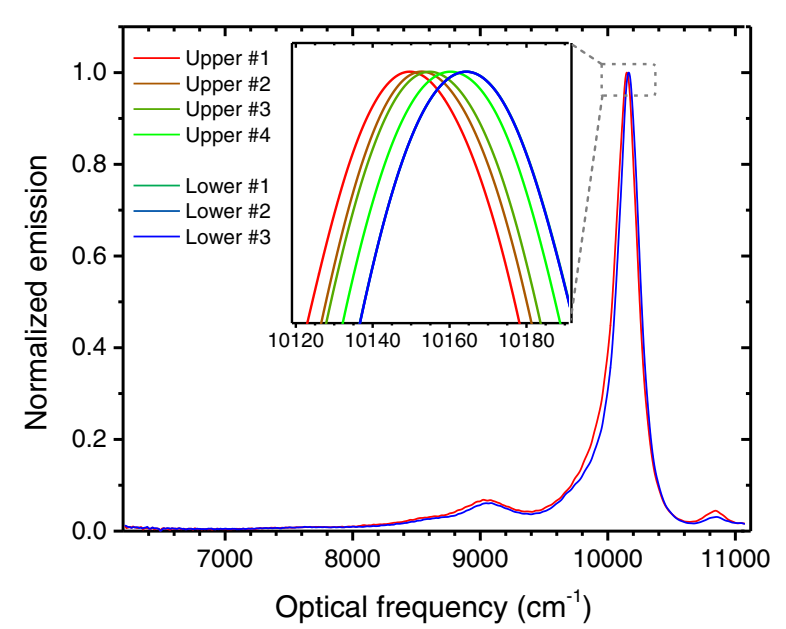

Figure 3. Normalized emission spectra (in quantal units) measured with 784 $\mathrm{nm}$ excitation for separated and diluted fractions extracted from the upper and lower bands shown in Figure 1. The three lower band spectra are too similar to be distinguished in the figure or the inset. 


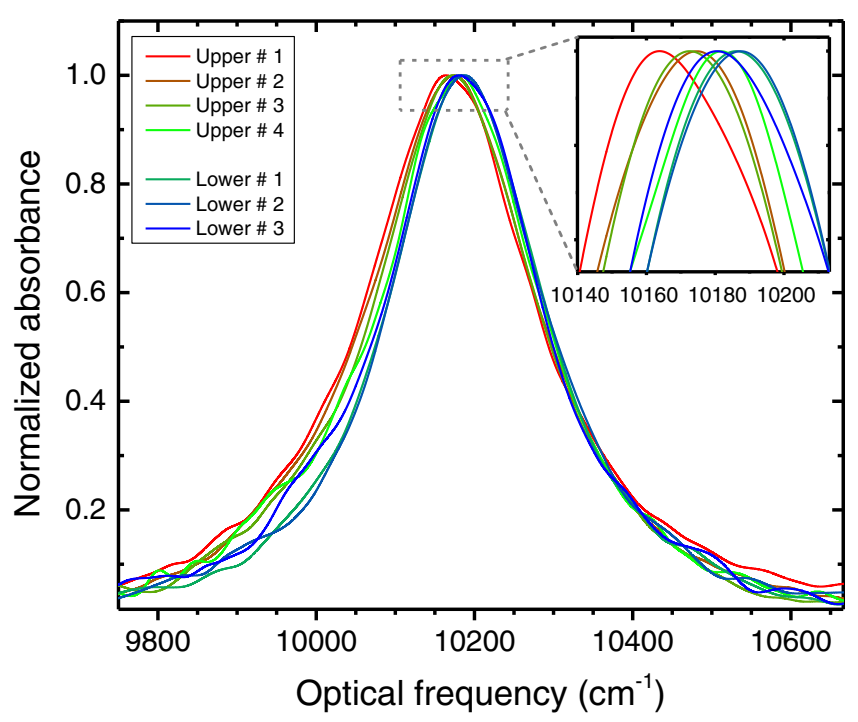

Figure 4. Normalized absorption spectra of the seven extracted and diluted fractions, after subtraction of a linear background.

Stokes shifts that were uniform within our experimental uncertainty. Similarly, we found no trend in peak width or Stokes shift values among the four upper band fractions. However, those upper band fractions did show emission peak positions that increased in frequency by $13 \mathrm{~cm}^{-1}$ from the top to the bottom layer. Very similar results were found from spectral analyses of fractions extracted from a second NDGU tube processed with the same protocol (see Figure S6). We also confirmed the trend in peak position by measuring variance spectra, in which the peak positions can differ from conventional spectra because of heavier weighting toward subpopulations with higher quantum yields. This occurs because the variance signals from different portions of an inhomogeneously broadened peak are inversely proportional to the number of particles emitting in that spectral portion. Our variance spectra of the four upper band fractions have peak positions that are ca. $5 \mathrm{~cm}^{-1}$ blue-shifted from those in the corresponding conventional emission spectra, but they confirm the same $13 \mathrm{~cm}^{-1}$ increase in peak positions from top to bottom fractions within the upper band and also confirm the absence of such a trend for the lower band (see Figure S10a).

Covariance analyses. - Variance data can be analyzed to construct 2D covariance plots, which reveal correlations between spectral signals at pairs of emission wavelengths. ${ }^{9}$ For example, if two emission features come from the same (homogeneous) subpopulation of nanotubes, then their intensities will be cross-correlated and an offdiagonal peak in the 2D covariance plot will appear. As demonstrated previously, ${ }^{9}$ slices through $2 \mathrm{D}$ covariance plots give the spectra of homogeneous subpopulations in heterogeneous samples. The upper panel of Figure 5 shows overlaid plots of the normalized covariance slice at the $(6,5)$ emission peak and the mean emission spectrum, both measured for the upper band fourth fraction. Although the two traces appear very similar, there are systematic differences between them that show inhomogeneous components in the mean spectrum. These are quantified in the mean minus covariance values plotted in the lower panel. In addition to the labeled peaks from minor $(9,1)$ and $(6,4)$ impurities (which are clearly inhomogeneous features), there are substantial differences on the low frequency side of the $E_{11}$ peak near the position of the $Y_{1}$ sideband. We suggest that the excess low frequency emission in the mean spectrum comes from a subpopulation of $(6,5)$ SWCNTs having slightly red-shifted emission peaks or an intensified $\mathrm{Y}_{1}$ side band, which has previously been assigned as a defect-induced or extrinsic feature. ${ }^{15}$ Note that the mean and covariance spectra are much better matched at the positions of the $G_{1}$ and $X_{1}$ side bands, which have been assigned as intrinsic features. ${ }^{15}$ The extended low

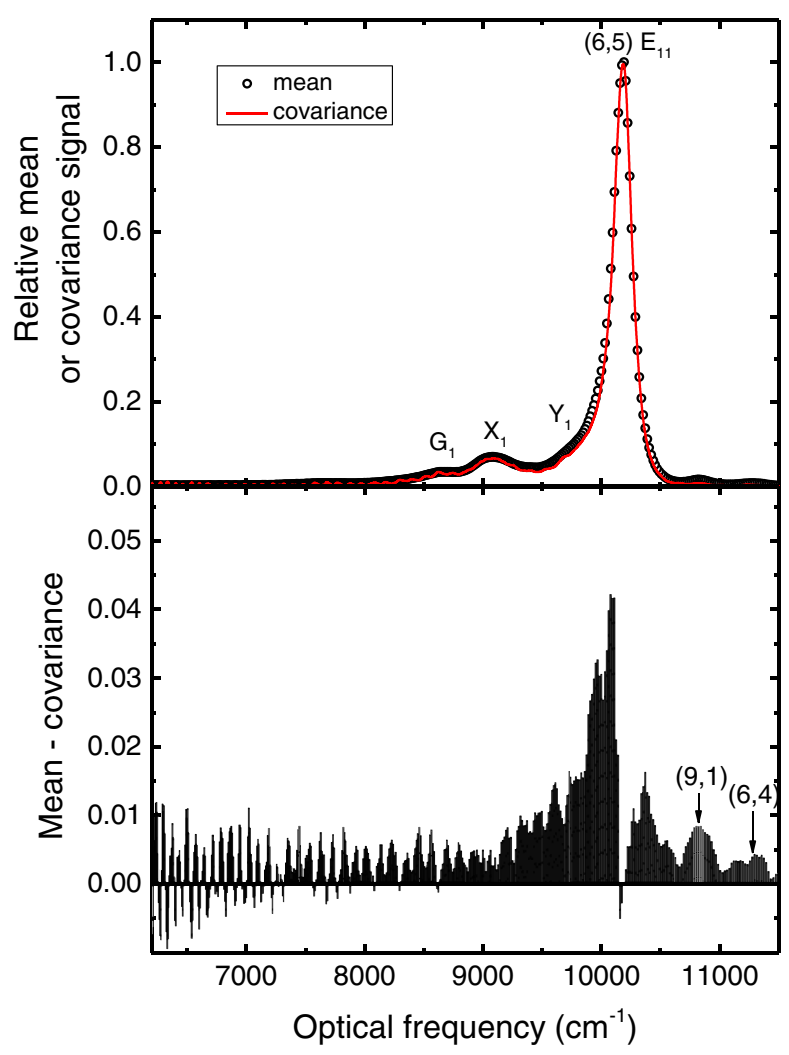

Figure 5. Top frame: Relative mean spectrum (open circles) and covariance spectrum (red curve) measured for the diluted fourth fraction of the upper NDGU band. Main and side band spectral features are marked. Bottom frame: Difference between the mean and covariance spectra in the upper frame, plotted on an expanded scale. Features attributed to minor $(9,1)$ and $(6,4)$ impurities are marked.

frequency wing in the mean spectrum makes its fwhm value exceed that of the covariance spectrum by $8 \mathrm{~cm}^{-1}$ (see Figure $\mathrm{S} 10 \mathrm{~b}$ ). We interpret this difference as ensemble inhomogeneity (spectral structure variations among nanotubes) and conclude that even nanotubes within a single fraction extracted from one NDGU band show measurable differences. These are separate from the apparent spectral inhomogeneity within individual SWCNTs that is deduced from the large Gaussian line shape component observed in single-nanotube emission spectra. ${ }^{15}$ We presume that such intra-particle inhomogeneity is induced by local differences associated with nanotube defects or nonuniform surfactant coverage.

Contour plots of 2D covariance data can allow convenient visualization of some spectral line shapes and inhomogeneities. Figure 6 displays an example of such a plot (with linear and logarithmic intensity scales) for the sample of Figure 5. The variance spectrum can be traced along the positive diagonal, which is also a reflection symmetry element for the plot. It shows, in addition to the major $(6,5)$ feature, smaller ones from $(6,4)$ and $(9,1)$ impurities. Horizontal slices are covariance spectra; the one plotted in Figure 5 passes through the $(6,5)$ peak. From the contour shapes and colors along that slice in the lower frame of Figure 6 , one can see the $\mathrm{X}_{1}$ side band and the low frequency tail of the $(6,5) \mathrm{E}_{11}$ peak. There are also minor features in that horizontal covariance slice at the $(6,4)$ and $(9,1)$ emission frequencies. However, these should not be associated with $(6,5)$ SWCNTs in a completely disaggregated sample. We assign these faint off-diagonal features, which have amplitudes several hundred times below the main peak, to a small population of clusters containing $(6,5)$ nanotubes loosely aggregated with the $(6,4)$ and $(9,1)$ impurities. ${ }^{9}$ These features are nearly invisible in the linearly scaled covariance plot or in the red curve of Figure 5.

In general, the Gaussian peak shapes associated with inhomogeneous broadening can be recognized in covariance $2 \mathrm{D}$ plots by their 

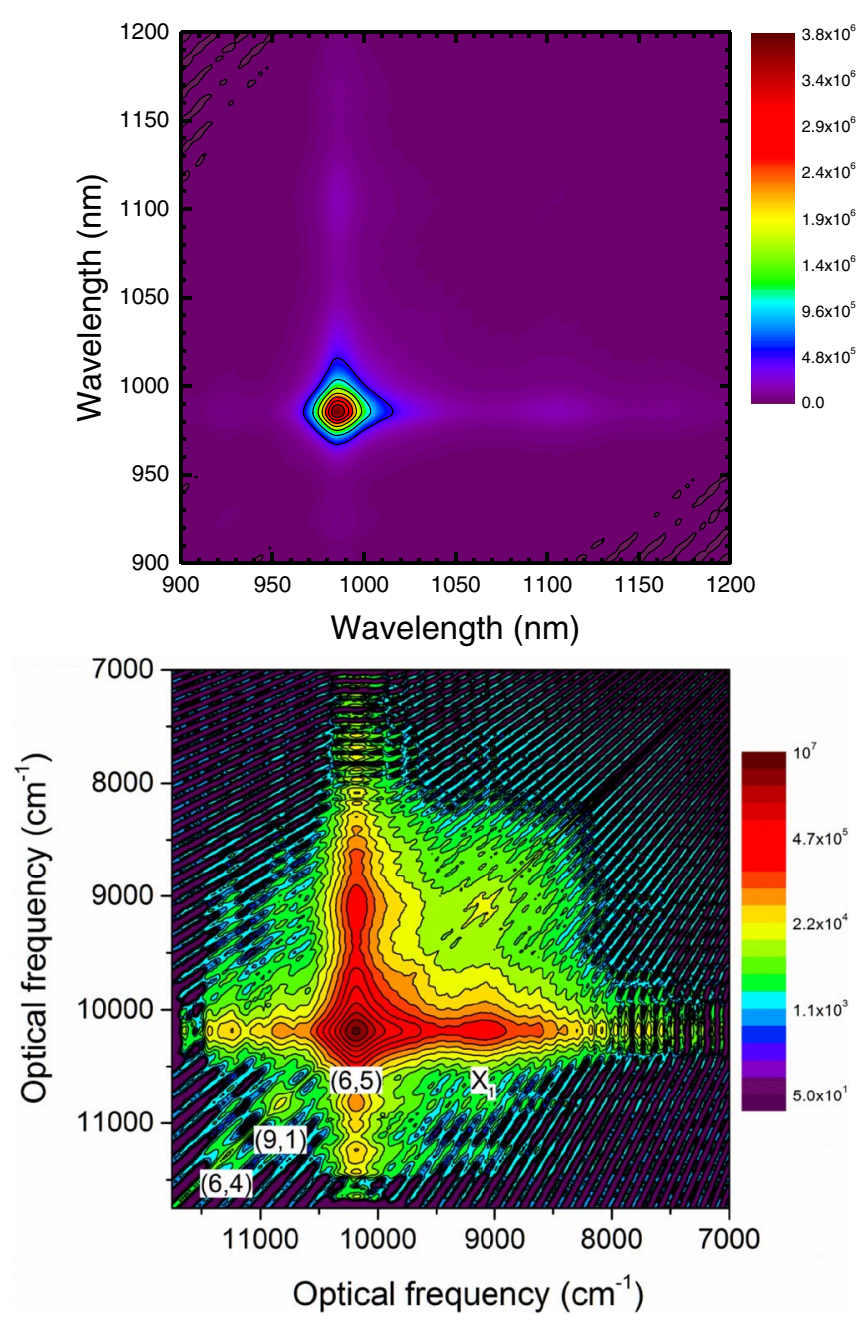

Figure 6. Covariance contour plots measured for the fourth fraction of the upper NDGU band. Intensity scales are linear in the top frame and logarithmic in the bottom frame. The main feature is from $(6,5)$ SWCNTs; weak features assigned to $(6,4)$ and $(9,1)$ impurities are also labeled.

rigorously circular contours. By contrast, Lorentzian peaks lead to highly star-shaped contours. Examples of these and related shapes are illustrated in the simulated covariance contour plots of Figures S12$\mathrm{S} 15$. The shape of the $(6,5) \mathrm{E}_{11}$ contour in the lower frame of Figure 6 is characteristic of a distorted Voigt (mixed Lorentzian and Gaussian) emission profile.

Quantum yields.-We have also measured absolute fluorescence quantum yields for the diluted extracted fractions. The results are plotted in Figure 7 as a function of depth in the centrifuge tube. Quantum yields in the upper band fractions ranged from 0.69 to $0.81 \%$, with no clear position dependence. However, the lower band quantum yields decreased from $0.93 \%$ for the top layer to $0.68 \%$ for the bottom. These values are very close to the $0.8 \%$ quantum yield reported for SC-suspended $(6,5)$ SWCNTs with average lengths matching our sample. ${ }^{20}$ A more recent study found lower quantum yields of only $0.2 \%$ for $(6,5)$ SWCNTs purified by gel chromatography and DGU but suspended in aqueous SDS, ${ }^{21}$ which is known to give weaker SWCNT emission than SC. ${ }^{22}$ To check the pattern in our quantum yield results, we made a set of independent measurements using variance spectroscopy. As described above, variance data provided for each fraction both the mean sample emission and the number of emitting particles. The ratio of these values gave relative emission per particle, which should be proportional to fluorescence quantum yield. Those findings are plotted as solid circles in Figure 7, where the simi-

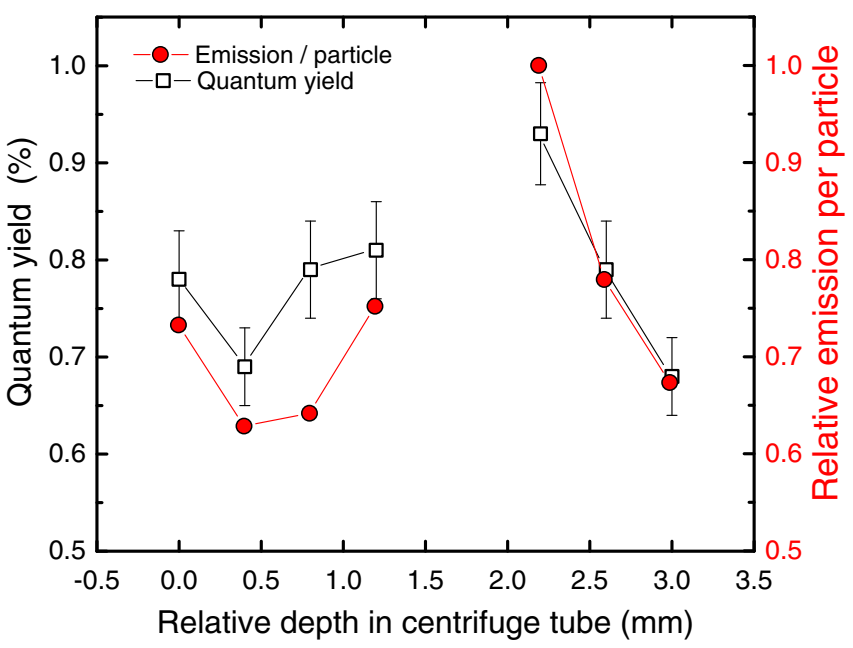

Figure 7. Overlaid plots of absolute fluorescence quantum yields (open squares) and relative emission per nanotube (solid circles) measured for the extracted and diluted fractions from the upper and lower NDGU bands.

larity to the quantum yield pattern is clear. We conclude that the layers within the lower band differ systematically in emissive quantum yield. To see if this variation was associated with differences in nanotube length, we measured LAND data for the top and bottom fractions of the lower band and found distributions that differed in width but had the same average value of $300 \mathrm{~nm}$ (see Figure S7). We suggest that the quantum yield variations within the lower band are more likely caused by differences in defect densities. This inhomogeneity was not evident from careful measurements of emission peak positions, widths, or Stokes shifts.

\section{Conclusions}

Although NDGU processing is considered one of the most refined methods for structurally sorting SWCNTs, we have found differences in spectral properties among separated NDGU fractions that were presumed to be equivalent. To observe these differences, one should first recognize that fluorescence spectra of NDGU layers measured through the walls of undisturbed centrifuge tubes may show significant spectral distortions from inner filter effect artifacts. In addition, the spectra of undiluted extracted fractions can be substantially broadened and shifted by nonuniform surfactant coverage. Both inner filter and coverage distortions can be removed by dilution with a suitably strong surfactant. Following such treatment, we found that one enantiomer band of $(6,5)$ SWCNTs shows emission peaks that blue-shift with increasing depth inside the band. In the other enantiomer band, emission spectra are uniform but the quantum yields vary with depth, ranging from 0.6 to $0.9 \%$. The source of these differences is currently unclear but may be uncovered in future studies. The recently developed method of variance spectroscopy confirmed the trends in quantum yields and provided covariance plots that sensitively revealed spectral variations among nanotubes within the same fraction of a single band. We presume that the spectral inhomogeneities found in this study must reflect a distribution of nanotube structural defects or surface functionalization. Therefore, investigators should be aware that $(n, m)$ - or enantiomerically-sorted SWCNT samples prepared by careful NDGU may still contain measurable structural and spectral nonuniformities.

\section{Acknowledgments}

This research has been supported by the National Science Foundation (grant CHE-1409698) and the Welch Foundation (grant C-0807). S. R. S is grateful to the National Science Foundation for support through an AGEP-GRS award (CHE-1549024). R. B. W. has a financial interest in Applied NanoFluorescence, LLC, which manufactures one of the instruments used in this study. 


\section{References}

1. S. Reich, C. Thomsen, and J. Maultzsch, Carbon Nanotubes: Basic Concepts and Physical Properties; Wiley-VCH: Weinheim, 2004.

2. S. Ghosh, S. M. Bachilo, and R. B. Weisman, Nat. Nanotechnol., 5, 443 (2010).

3. S. Cambre and W. Wenseleers, Angewandte Chemie - International Edition, 50, 2764 (2011).

4. A. A. Green, M. C. Duch, and M. C. Hersam, Nano Res., 2, 69 (2009).

5. F. Wang, K. Matsuda, A. F. M. M. Rahman, X. Peng, T. Kimura, and N. Komatsu, J. Am. Chem. Soc., 132, 10876 (2010).

6. J. A. Fagan, J. Y. Huh, J. R. Simpson, J. L. Blackburn, J. M. Holt, B. A. Larsen, and A. R. H. Walker, ACS Nano, 5, 3943 (2011).

7. J.-D. R. Rocha, S. M. Bachilo, S. Ghosh, S. Arepalli, and R. B. Weisman, Anal. Chem., 83, 7431 (2011).

8. R. B. Weisman, Anal. Bioanal. Chem., 396, 1015 (2010).

9. J. K. Streit, S. M. Bachilo, S. R. Sanchez, C.-W. Lin, and R. B. Weisman, J. Phys. Chem. Lett., 3976 (2015).

10. M. Magg, Y. Kadria-Vili, P. Oulevey, R. B. Weisman, and T. Bürgi, J. Phys. Chem. Lett., 7, 221 (2016).

11. Y. Kadria-Vili, G. Canning, S. M. Bachilo, and R. B. Weisman, Anal. Chem., 86, 11018 (2014).
12. Y. Kadria-Vili, S. M. Bachilo, and R. B. Weisman, in preparation,

13. J. K. Streit, S. M. Bachilo, A. V. Naumov, C. Khripin, M. Zheng, and R. B. Weisman, ACS Nano, 6, 8424 (2012).

14. S. R. Sanchez, S. M. Bachilo, Y. Kadria-Vili, C.-W. Lin, and R. B. Weisman, Nano Lett, 16, 6903 (2016).

15. Y. Kadria-Vili, S. M. Bachilo, J. L. Blackburn, and R. B. Weisman, J. Phys. Chem. C, 120, 23898 (2016).

16. S. M. Bachilo, M. S. Strano, C. Kittrell, R. H. Hauge, R. E. Smalley, and R. B. Weisman, Science, 298, 2361 (2002)

17. R. B. Weisman and S. M. Bachilo, Nano Lett, 3, 1235 (2003).

18. S. Ghosh, S. M. Bachilo, and R. B. Weisman, Fullerenes, Nanotubes, Carbon Nanostruct., 22, 269 (2014).

19. J. R. Lakowicz, Principles of Fluorescence Spectroscopy; 2nd ed.; Kluwer Academic: New York, 1999.

20. T. Hertel, S. Himmelein, T. Ackermann, D. Stich, and J. Crochet, ACS Nano, 4, 7161 (2010).

21. A. Graf, Y.Zakharko, S. P. Schießl, C. Backes, M. Pfohl, B. S. Flavel, and J. Zaumseil, Carbon, 105, 593 (2016).

22. F. Bonaccorso, T. Hasan, P. H. Tan, C. Sciascia, G. Privitera, G. Di Marco, P. G. Gucciardi, and A. C. Ferrari, J. Phys. Chem. C, 114, 17267 (2010). 